UM-P-93/51

OZ-93/11

hep-ph/9308256

\title{
Study of the simplest realistic Higgs sector in quark-lepton symmetric models
}

\author{
Y. Levin' and R. R. Volkasf \\ Research Centre for High Energy Physics, School of Physics, \\ University of Melbourne, Parkville 3052, Australia
}

\begin{abstract}
A discrete symmetry between quarks and (generalized) leptons can exist in nature, and its spontaneous symmetry breaking scale can be as low as a few TeV. Such a discrete symmetry also has interesting implications for how electroweak symmetry is spontaneously broken, because the simplest version of the theory requires two electroweak Higgs doublets rather than one in order to provide acceptable values for quark and lepton masses. The effective theory generated at electroweak-scale energies is thus a particular type of two-Higgs-doublet model. We point out in this paper that the broken discrete symmetry imposes very interesting constraints on the form of the Yukawa couplings between physical Higgs bosons and quarks and leptons. In particular, we find that the flavour-changing neutral Higgs couplings to down-sector quarks are proportional to the neutrino Dirac mass matrix. If neutrinos are Dirac particles, then the severe experimental upper bounds on their mass values renders tree-level neutral flavour-changing Higgs effects on down-quark systems like $K^{0}-\bar{K}^{0}$ negligibly small. We also discuss minimization of some relevant Higgs potentials and some other pertinent phenomenological issues.
\end{abstract}

\footnotetext{
${ }^{1}$ email: yal@tauon.ph.unimelb.edu.au

${ }^{2}$ email: U6409503@hermes.ucs.unimelb.edu.au
} 


\section{Introduction}

If there is physics beyond the Standard Model (SM), then it probably involves at least one new symmetry principle of nature. Our experience with the interactions of quarks and leptons strongly suggests that the search for new symmetries is likely to bear fruit, because symmetries play a central role in the SM.

If we adopt a "bottom-up" approach to model-building - that is, if our starting point is what we know of low-energy particle interactions rather than an ambitious unifying principle of some sort - then the first new invariance we might hope to uncover at some energy scale $>100 \mathrm{GeV}$ is likely to be a discrete symmetry. This is a reasonable suggestion simply because discrete symmetries are the simplest candidates. For instance, one may like to suppose that the complete Lagrangian of the world, describing some fundamental unified theory, displays some large, elegant, continuous invariance group which is broken in many stages down to $G_{S M}=$ $\mathrm{SU}(3)_{c} \otimes \mathrm{SU}(2)_{L} \otimes \mathrm{U}(1)_{Y}$ and finally just $\mathrm{SU}(3)_{c} \otimes \mathrm{U}(1)_{Q}$. It could well be that the first enlargement of the symmetry group of nature above the electroweak scale involves some discrete symmetry subgroup of the large fundamental invariance group. Discrete subgroups might well survive intact down to quite low energies because they yield less new physics than either global or local continuous symmetries, and are thus likely to be less phenomenologically constrained. Alternatively, it may turn out that discrete symmetries are of greater fundamental importance than current theoretical prejudices allow.

If we look at a quark-lepton generation,

$$
\begin{aligned}
& Q_{L} \sim(3,2)(1 / 3), \quad u_{R} \sim(3,1)(4 / 3), \quad d_{R} \sim(3,1)(-2 / 3) \\
& \ell_{L} \sim(1,2)(-1), \quad e_{R} \sim(1,1)(-2), \quad\left[\nu_{R} \sim(1,1)(0)\right],
\end{aligned}
$$

where the quantum numbers are given with respect to $G_{S M}$, then three generic classes of discrete symmetries suggest themselves: (i) horizontal, (ii) left-right and (iii) quark-lepton symmetries. Horizontal symmetries are the simplest to implement in the sense that no extension to the gauge group $G_{S M}$ is mandatory. Left-right symmetry (either parity or charge-conjugation invariance) can be implemented if we extend $G_{S M}$ to the left-right group $G_{L R}=\mathrm{SU}(3)_{c} \otimes \mathrm{SU}(2)_{L} \otimes \mathrm{SU}(2)_{R} \otimes \mathrm{U}(1)_{B-L}$. This gauge group extension requires the inclusion of a new fermion - the right-handed neutrino - whose presence is optional in the SM. Quark-lepton discrete symmetry (q- $\ell$ symmetry for short) can be implemented if we extend $G_{S M}$ to the new group $G_{q \ell}$ where

$$
G_{q \ell}=\mathrm{SU}(3)_{\ell} \otimes \mathrm{SU}(3)_{q} \otimes \mathrm{SU}(2)_{L} \otimes \mathrm{U}(1)_{X},
$$


where $\mathrm{SU}(3)_{\ell}$ is a "leptonic colour" group and $\mathrm{SU}(3)_{q}$ is just the usual colour group with a new name [1]. This gauge group extension also requires the introduction of new fermions, in this case the leptonic colour partners of standard leptons (as well as a right-handed neutrino).

Horizontal symmetries, discrete or otherwise, and left-right symmetry have been assiduously studied for the past twenty years or so, and they remain very important and interesting possibilities for new physics. The possible existence of a quark-lepton discrete symmetry has, however, only been pointed out quite recently, and so much work remains to be done in this area. Actually, some work has already been performed on neutral current phenomenology [2], partial unification schemes [3], the fermion mass problem [4] and cosmological implications [5, 6]. However, two important aspects of $\mathrm{q}-\ell$ symmetric models require more attention in the literature. The first area concerns the phenomenology of the new strongly-interacting sector predicted by the theory. [An $\mathrm{SU}(2)$ subgroup of leptonic colour remains unbroken and confines the exotic partners of the standard leptons into unstable, non-relativistic bound states.] Although some initial studies were conducted in Refs. [6, 7], much more detailed work is required. The other area is the subject of this paper: the phenomenology of the extended Higgs sector of $q-\ell$ symmetric models.

Quark-lepton symmetric models can employ a number of different types of Higgs sectors, depending on what one wants to do exactly. For instance, if one wishes to employ the see-saw mechanism [8] for neutrino masses then a more complicated Higgs sector is required than if one just fine-tunes small neutrino masses. Also, the $\mathrm{q}-\ell$ discrete symmetry can induce troublesome mass relations between quarks and leptons if the Higgs sector is too simple. It is possible to adopt different attitudes to what one should do about this problem, and this leads to different Higgs physics. There is no clearly preferred option for the Higgs sector at the moment. In this paper we will therefore review the major possibilities, but will ultimately concentrate on the detailed phenomenology of a particular concrete scenario for reasons we will make clear later.

The rest of this paper is structured as follows: In Sec. II we review the possible choices for Higgs sectors in $q-\ell$ symmetric models. Section III forms the core of our paper. We study one simple and workable scenario in detail. We look at (i) the construction and minimization of Higgs potentials, (ii) the identification of the physical Higgs fields and their Yukawa couplings, and (iii) the derivation of bounds from tree-level flavour-changing effects induced by the neutral Higgs bosons. Our concluding discussion forms Sec. IV. 


\section{Higgs sectors for quark-lepton symmetric models.}

The simplest gauge group which supports discrete $\mathrm{q}-\ell$ symmetry is given by $G_{q \ell}$ in Eq. (目). A fermionic generation is given by

$$
\begin{aligned}
Q_{L} \sim(1,3,2)(1 / 3), & u_{R} \sim(1,3,1)(4 / 3), \quad d_{R} \sim(1,3,1)(-2 / 3), \\
F_{L} \sim(3,1,2)(-1 / 3), & E_{R} \sim(3,1,1)(-4 / 3), \quad N_{R} \sim(3,1,1)(2 / 3),
\end{aligned}
$$

where the standard leptons $\ell_{L}, e_{R}$ and $\nu_{R}$ are one of the colour components of $F_{L}, E_{R}$ and $N_{R}$, respectively. This gauge structure can clearly support a discrete symmetry between quarks and the generalized leptons. The most straightforward possibility is the symmetry

$$
Q_{L} \leftrightarrow F_{L}, \quad u_{R} \leftrightarrow E_{R}, \quad d_{R} \leftrightarrow N_{R}, \quad G_{q}^{\mu} \leftrightarrow G_{\ell}^{\mu}, \quad W^{\mu} \leftrightarrow W^{\mu}, \quad C^{\mu} \leftrightarrow-C^{\mu},
$$

where $G_{q}^{\mu}, G_{\ell}^{\mu}, W^{\mu}$ and $C^{\mu}$ are the gauge bosons of $\mathrm{SU}(3)_{q}, \mathrm{SU}(3)_{\ell}, \mathrm{SU}(2)_{L}$ and $\mathrm{U}(1)_{X}$ respectively. Other varieties are also possible (see Ref. [9] for a complete discussion), but for definiteness we will concentrate on this form of discrete $q-\ell$ symmetry in this paper.

The standard model is recovered as an effective low-energy theory through the two-stage symmetry breaking chain

$$
G_{q \ell} \rightarrow \mathrm{SU}(2)^{\prime} \otimes G_{S M} \rightarrow \mathrm{SU}(2)^{\prime} \otimes \mathrm{SU}(3)_{q} \otimes \mathrm{U}(1)_{Q},
$$

where $\mathrm{SU}(2)^{\prime}$ is an unbroken subgroup of leptonic colour $\mathrm{SU}(3)_{\ell}$. The discrete $\mathrm{q}-\ell$ symmetry is broken at the same time as leptonic colour in the simplest scenarios. The weak hypercharge generator $Y$ of $G_{S M}$ is given by

$$
Y=X+T / 3
$$

where $T \equiv \operatorname{diag}(-2,1,1)$ is one of the diagonal generators of leptonic colour. Each standard lepton has a pair of exotic partners through leptonic colour invariance. After the first stage of symmetry breaking - that is, $G_{q \ell} \rightarrow \mathrm{SU}(2)^{\prime} \otimes G_{S M}$ - the standard leptons are identified as the $T=-2$ components of the leptonic colour triplets, while the $T=1$ components form an $\mathrm{SU}(2)^{\prime}$ doublet of exotic fermions with electric-charge $\pm 1 / 2$ called "liptons." All of the particles which feel the residual $\mathrm{SU}(2)^{\prime}$ force can be made heavy, apart from the gauge bosons of $\mathrm{SU}(2)^{\prime}$. Therefore, although the unbroken gauge group at the electroweak scale is larger than $G_{S M}$, the effective theory at this scale is still the SM. 


\subsection{Higgs sector A}

The simplest way that the symmetry breaking chain of Eq. (5) can be induced is by choosing the Higgs sector,

$$
\chi_{1} \sim(1,3,1)(-2 / 3), \quad \chi_{2} \sim(3,1,1)(2 / 3), \quad \phi \sim(1,2,1)(1)
$$

where $\chi_{1} \leftrightarrow \chi_{2}$ and $\phi \leftrightarrow \phi^{c} \equiv i \tau_{2} \phi^{*}$ under q- $\ell$ symmetry. Under the subgroup $\mathrm{SU}(2)^{\prime} \otimes G_{S M}$ the transformation law for $\chi_{2}$ is

$$
\begin{aligned}
& \chi_{2} \rightarrow(1,1,1)(0) \oplus(2,1,1)(1), \\
& \chi_{2} \rightarrow \chi_{2}^{0} \oplus \tilde{\chi}_{2},
\end{aligned}
$$

where the second line establishes our nomenclature for the component fields under the subgroup. Therefore the first stage of symmetry breaking is induced by the vacuum expectation value (VEV) pattern,

$$
\left\langle\chi_{1}\right\rangle=0, \quad\left\langle\chi_{2}^{0}\right\rangle=v \neq 0, \quad\left\langle\tilde{\chi}_{2}\right\rangle=0, \quad\langle\phi\rangle=0,
$$

while the second stage (that is, standard electroweak breaking) is induced by $\langle\phi\rangle=$ $u \neq 0$. The hierarchy $v \gg u$ is required in order to satisfy phenomenological bounds.

All of the Higgs multiplets in Eq. (17) couple to fermions through Yukawa interactions. The Lagrangian is

$$
\begin{aligned}
\mathcal{L}_{\text {Yuk }} & =h_{1}\left[\overline{\left(Q_{L}\right)^{c}} Q_{L} \chi_{1}+\overline{\left(F_{L}\right)^{c}} F_{L} \chi_{2}\right]+h_{2}\left[\overline{\left(u_{R}\right)^{c}} d_{R} \chi_{1}+\overline{\left(E_{R}\right)^{c}} N_{R} \chi_{2}\right] \\
& +\lambda_{1}\left[\bar{Q}_{L} d_{R} \phi+\bar{F}_{L} N_{R} \phi^{c}\right]+\lambda_{2}\left[\bar{Q}_{L} u_{R} \phi^{c}+\bar{F}_{L} E_{R} \phi\right]+\text { H.c. }
\end{aligned}
$$

After the first stage of symmetry breaking, liptons gain masses $h_{1} v$ and $h_{2} v$ from these Yukawa coupling terms. Since $v \gg u$ this means that the liptons will in general be much more massive than standard leptons and quarks, provided that the Yukawa coupling constants $h_{1,2}$ are not extremely small. The standard fermions of course gain masses after the second stage of symmetry breaking from the usual $\phi$ Yukawa interactions. Note, however, that the discrete $q-\ell$ symmetry imposes the tree-level mass relations

$$
m_{u}=m_{e}, \quad m_{d}=m_{\nu}^{\text {Dirac }}
$$

which are not phenomenologically acceptable. Radiative corrections will alter these mass relations, but not enough to make them tenable.

\footnotetext{
${ }^{3}$ Note that an SU(3) transformation can be used to bring any VEV for $\chi_{2}$ into the $\left\langle\chi_{2}^{0}\right\rangle=v \neq 0$, $\left\langle\tilde{\chi}_{2}\right\rangle=0$ form.
} 
We therefore see that the symmetry breaking pattern we desire can be induced by the simple Higgs sector of Eq. (7). This scheme has the desirable property that liptons are in general expected to be much heavier than leptons and quarks. However, it has the undesirable mass relations of Eq. (11). The next Higgs sector we examine retains the desirable features of this prototype but improves on the unsuccessful mass relations.

\subsection{Higgs sector $\mathrm{B}$}

\subsubsection{Introduction}

The amended Higgs sector consists of $\chi_{1}, \chi_{2}$ plus two electroweak Higgs doublets

$$
\phi_{1} \sim(1,1,2)(1)=\left(\begin{array}{c}
\phi_{1}^{+} \\
\phi_{1}^{0}
\end{array}\right) \quad \text { and } \quad \phi_{2} \sim(1,1,2)(-1)=\left(\begin{array}{c}
\phi_{2}^{0} \\
\phi_{2}^{-}
\end{array}\right)
$$

which interchange under the $q-\ell$ discrete symmetry. The symmetry breaking pattern is the same as in Eq. (5), with both electroweak doublets in general participating in breaking the electroweak symmetry: $\left\langle\phi_{1}\right\rangle=u_{1}$ and $\left\langle\phi_{2}\right\rangle=u_{2}$.

The Yukawa Lagrangian for the $\chi$ multiplets is obviously the same as for Higgs sector A, but the $\phi$ interactions are different. There are actually two different models which use Higgs sector B. These models are distinguished by the way the charge conjugates of $\phi_{1}$ and $\phi_{2}$ behave under $q-\ell$ discrete symmetry. This in turn leads to two different electroweak Yukawa Lagrangians and also to two different Higgs potentials. The existence of two models using Higgs sector B is a subtle point which was overlooked in previous papers [2].

\subsubsection{Model 1}

Consider the charge conjugate Higgs fields $\phi_{1}^{c}$ and $\phi_{2}^{c}$ where

$$
\phi_{1}^{c} \equiv i \tau_{2} \phi_{1}^{*}=\left(\begin{array}{c}
\phi_{1}^{0 *} \\
-\phi_{1}^{-}
\end{array}\right) \quad \text { and } \quad \phi_{2}^{c} \equiv i \tau_{2} \phi_{2}^{*}=\left(\begin{array}{c}
\phi_{2}^{+} \\
-\phi_{2}^{0 *}
\end{array}\right)
$$

In Model 1 , the action of the $\mathrm{q}-\ell$ discrete symmetry on the Higgs doublets is

$$
\phi_{1} \leftrightarrow \phi_{2}, \quad \text { and } \quad \phi_{1}^{c} \leftrightarrow \phi_{2}^{c},
$$


which in terms of weak-isospin components has to be interpreted to mean that components of like weak-isospin interchange:

$$
\phi_{1}^{+} \leftrightarrow \phi_{2}^{0}, \quad \phi_{1}^{0} \leftrightarrow \phi_{2}^{-}, \quad \phi_{1}^{0 *} \leftrightarrow \phi_{2}^{+}, \quad \phi_{1}^{-} \leftrightarrow \phi_{2}^{0 *}
$$

This last set of interchanges commutes with complex conjugation, which is a necessary condition to maintain the invariance of the kinetic energy terms for $\phi_{1,2}$. The alternative possibility that unlike weak-isospin components interchange is not tenable, because of the minus signs appearing in the definitions of the charge conjugate doublets. For instance, $\phi_{1}^{0} \leftrightarrow \phi_{2}^{0}$ would have to be accompanied by $\phi_{1}^{0 *} \leftrightarrow-\phi_{2}^{0 *}$ according to Eqs. (13) and (14). Since this does not preserve invariance of the kinetic energy Lagrangian, this is not an allowed discrete symmetry and so we must interpret Eq. (14) as implying Eq. (15) at the weak-isospin component level. We similarly interpret the fermionic transformation $Q_{L} \leftrightarrow F_{L}$ as implying

$$
u_{L} \leftrightarrow N_{L} \quad \text { and } \quad d_{L} \leftrightarrow E_{L}
$$

Model 1 therefore has the curious feature that the left- and right-handed components of the quarks and leptons interchange in exactly opposite ways, as can be seen by comparing Eqs. (16) and (4). Another way to put this is that the Model 1 discrete symmetry is chiral.

The Yukawa interactions for Model 1 are given by

$$
\begin{aligned}
\mathcal{L}_{\text {Yuk }} & =\Lambda_{1}\left[\bar{Q}_{L} d_{R} \phi_{1}+\bar{F}_{L} N_{R} \phi_{2}\right]+\Lambda_{1}^{\prime}\left[\bar{Q}_{L} d_{R} \phi_{2}^{c}+\bar{F}_{L} N_{R} \phi_{1}^{c}\right] \\
& +\Lambda_{2}\left[\bar{Q}_{L} u_{R} \phi_{1}^{c}+\bar{F}_{L} E_{R} \phi_{2}^{c}\right]+\Lambda_{2}^{\prime}\left[\bar{Q}_{L} u_{R} \phi_{2}+\bar{F}_{L} E_{R} \phi_{1}\right]+\text { H.c. }
\end{aligned}
$$

The quark and lepton mass matrices are thusf

$$
\begin{array}{cc}
m_{u}=\Lambda_{2} u_{1}^{*}+\Lambda_{2}^{\prime} u_{2}, & m_{e}=-\Lambda_{2} u_{2}^{*}+\Lambda_{2}^{\prime} u_{1} \\
m_{d}=\Lambda_{1} u_{1}-\Lambda_{1}^{\prime} u_{2}^{*}, & m_{\nu}^{\text {Dirac }}=\Lambda_{1} u_{2}+\Lambda_{1}^{\prime} u_{1}^{*},
\end{array}
$$

and we see that the unsuccessful mass relations that are unavoidable with Higgs sector A do not apply in general. It is actually interesting to note that the relations $m_{u}=m_{e}$ and $m_{d}=m_{\nu}$ hold for all choices of the $\Lambda$ 's only if $u_{1}=u_{2}=0$, that is, when the electroweak symmetry is unbroken. This is consistent with Eq.(15) because we see that $\mathrm{q}-\ell$ discrete symmetry interchanges neutral Higgs bosons with charged

\footnotetext{
${ }^{4}$ After the first stage of symmetry breaking, the lipton partners of the standard leptons become heavy, and they will play no role in the rest of this paper. We will alternate between the notation $e$ and $E$, and between $\nu$ and $N$ whenever we find it convenient.
} 
Higgs bosons. Therefore, the electroweak symmetry breaking VEVs $u_{1}$ and $u_{2}$ for the neutral Higgs bosons necessarily also break the discrete symmetry, and this is why the tree-level fermionic mass relations are necessarily violated in this model.f Our previous observation that the left- and right-handed projections of quarks and leptons transform in exactly opposite ways is of course also consistent with the necessary violation of the fermionic mass relations. [We will see that Higgs sector A is similar to Model 2 to be presented below, in that the electroweak symmetry breaking VEV('s) do not inevitably also break q- $\ell$ symmetry.]

\subsubsection{Model 2}

Model 2 is defined to obey the discrete symmetry

$$
\phi_{1} \leftrightarrow \phi_{2}, \quad \text { and } \quad \phi_{1}^{c} \leftrightarrow-\phi_{2}^{c},
$$

which in component form has to mean that

$$
\phi_{1}^{+} \leftrightarrow \phi_{2}^{-}, \quad \phi_{1}^{0} \leftrightarrow \phi_{2}^{0}, \quad \phi_{1}^{-} \leftrightarrow \phi_{2}^{+}, \quad \phi_{1}^{0 *} \leftrightarrow \phi_{2}^{0 *} .
$$

By contrast to Model 1, the components of unlike weak-isospin interchange here. Note, of course, that the transformations in Eq. (20) commute with complex conjugation and thus leave the kinetic energy terms invariant. Similarly, the $Q_{L} \leftrightarrow F_{L}$ interchange is interpreted to mean

$$
u_{L} \leftrightarrow E_{L} \quad \text { and } \quad d_{L} \leftrightarrow N_{L}
$$

in Model 2. By contrast to Model 1 therefore, left- and right-handed projections of the fermions transform in identical ways under the discrete symmetry (in other words the discrete symmetry is vector-like).

\footnotetext{
${ }^{5}$ Of course, the discrete symmetry is dominantly broken by the VEV for $\chi_{2}$. The discrete symmetry breaking we are talking about in this section is an additional contribution which comes from the $\phi$ bosons.

${ }^{6}$ There is an alternative way to explain the discrete symmetry used in Model 2. We present it here because it makes no explicit reference to weak-isospin components, and thus should help clarify what we mean by the above symmetry. The symmetry is equivalent to $\phi_{1} \rightarrow \tau_{1} \phi_{2}, F_{L} \rightarrow \tau_{1} Q_{L}$, $C^{\mu} \rightarrow-C^{\mu}$ and $\tau_{i} W_{i}^{\mu} \rightarrow \tau_{1} \tau_{i} W_{i}^{\mu} \tau_{1}$, where $\tau_{1}$ is the first Pauli matrix. Under these transformations, $D^{\mu} \phi_{1} \rightarrow \tau_{1} D^{\mu} \phi_{2}$ where $D^{\mu}$ is the gauge-covariant derivative. Therefore the gauge-kinetic-energy terms for the two Higgs doublets interchange under the action of the symmetry. One can easily check that the gauge-invariant kinetic-energy terms for the fermions and for the gauge bosons are also invariant. This establishes that the discrete symmetry of Model 2 is well-defined. The $\tau_{1}$ matrix effectively tells us that components of unlike weak-isospin transform into each other here. Note also that the $W$-boson transformation above equates to $W_{3}^{\mu} \rightarrow-W_{3}^{\mu}$ and $W^{+\mu} \rightarrow W^{-\mu}$ at the component level. We thank H. Lew for alerting us to this.
} 
The Yukawa Lagrangian for Model 2 is obtained from that of Model 1 by the substitution $\phi_{2}^{c} \rightarrow-\phi_{2}^{c}$ :

$$
\begin{aligned}
\mathcal{L}_{\mathrm{Yuk}} & =\Lambda_{1}\left[\bar{Q}_{L} d_{R} \phi_{1}+\bar{F}_{L} N_{R} \phi_{2}\right]+\Lambda_{1}^{\prime}\left[-\bar{Q}_{L} d_{R} \phi_{2}^{c}+\bar{F}_{L} N_{R} \phi_{1}^{c}\right] \\
& +\Lambda_{2}\left[\bar{Q}_{L} u_{R} \phi_{1}^{c}-\bar{F}_{L} E_{R} \phi_{2}^{c}\right]+\Lambda_{2}^{\prime}\left[\bar{Q}_{L} u_{R} \phi_{2}+\bar{F}_{L} E_{R} \phi_{1}\right]+\text { H.c. }
\end{aligned}
$$

The quark and lepton mass matrices are thus

$$
\begin{array}{cc}
m_{u}=\Lambda_{2} u_{1}^{*}+\Lambda_{2}^{\prime} u_{2}, & m_{e}=\Lambda_{2} u_{2}^{*}+\Lambda_{2}^{\prime} u_{1} \\
m_{d}=\Lambda_{1} u_{1}+\Lambda_{1}^{\prime} u_{2}^{*}, & m_{\nu}^{\text {Dirac }}=\Lambda_{1} u_{2}+\Lambda_{1}^{\prime} u_{1}^{*} .
\end{array}
$$

Once again, the bad mass relations of Eq. (11) are in general violated. Note, however, that the mass relations will be reinstated in Model 2 if $u_{1}=u_{2} \neq 0$. This is consistent with the transformation laws in Eq. (20) since the neutral Higgs bosons interchange. If the two VEVs are equal, then discrete $q-\ell$ symmetry is clearly not broken during electroweak symmetry breaking, and so the tree-level fermionic mass relations ensue.

For the sake of completeness we note that Higgs sector A behaves in a similar way to Model 2. The transformation $\phi \leftrightarrow \phi^{c}$ has to be interpreted as implying $\phi^{0} \leftrightarrow \phi^{0 *}$ and $\phi^{+} \leftrightarrow-\phi^{-}$. Since the phase of the VEV $u$ is unobservable, $q-\ell$ symmetry is not broken during electroweak symmetry breaking and so the bad mass relations follow.

\subsubsection{Discussion}

Although both Models 1 and 2 using Higgs sector B have no mass relation problem, they also have no predictive power for masses. This is perhaps unfortunate, since we would prefer to have an enlarged symmetry like discrete $q-\ell$ symmetry actually increase the predictive power for the masses of quarks and leptons. Higgs sector A is predictive, but the predictions are wrong. Another response to the problem posed by Higgs sector A would therefore be to look for some other modification of it which maintained its predictive power for masses but this time with correct predictions. No such modification is known at present, but its desirability motivates that an on-going search be maintained.

Because the use of Higgs sector B is the simplest way to avoid the fermion mass relation problem, it will be the principal focus of study in this paper (see Sec.III). The main new qualitative result to be presented is that discrete $q-\ell$ symmetry continues to furnish us with more predictive power, even when all trace of it has disappeared from the fermion mass spectrum. We will see that quark-lepton partnership in both Models 1 and 2 is manifested in the Yukawa interactions between fermions and 
physical Higgs bosons. In particular, we will see that the flavour-changing neutral Higgs-boson term for a given fermion is proportional to the mass matrix of its partner under the discrete symmetry.

\subsection{Other Higgs sectors}

There are several other interesting Higgs sectors one can use. For instance if one wishes to address the issue of small neutrino masses, one may introduce a see-saw mechanism [8] by the introduction of the Higgs multiplets [1],

$$
\Delta_{1} \sim(1, \overline{6}, 1)(4 / 3) \text { and } \Delta_{2} \sim(\overline{6}, 1,1)(-4 / 3),
$$

where $\Delta_{1}$ and $\Delta_{2}$ interchange under q- $\ell$ symmetry. A large VEV for the neutral component of $\Delta_{2}$ induces large Majorana masses for the right-handed neutrinos, thus producing the see-saw phenomenon. Either Higgs sector A or Higgs sector $\mathrm{B}$ can be augmented by the introduction of these antisextets, and we will call the resulting Higgs sector generically as "Higgs sector C."

Another Higgs field of some interest is a real gauge singlet $\sigma$ which is odd under q- $\ell$ symmetry $(\sigma \rightarrow-\sigma)$. The purpose of $\sigma$ would be to separate the scales of leptonic colour and discrete symmetry breaking. A motivation for this might be cosmology, because such a scenario allows one to break the discrete symmetry before an inflationary epoch in the Hot Big Bang picture, while leptonic colour could be broken after inflation. This can be used to render innocuous the cosmological domain walls formed during the $q-\ell$ symmetry breaking phase transition, while retaining exact leptonic colour down to TeV-scale energies [5]. Any of Higgs sectors A, B or $\mathrm{C}$ can extended by introducing $\sigma$.

Finally, we comment that the gauge group $G_{q \ell}$ is but the simplest symmetry which supports a discrete q- $\ell$ symmetry. An interesting extension is provided by the gauge group $G_{q \ell L R}$ where

$$
G_{q \ell L R}=\mathrm{SU}(3)_{\ell} \otimes \mathrm{SU}(3)_{q} \otimes \mathrm{SU}(2)_{L} \otimes \mathrm{SU}(2)_{R} \otimes \mathrm{U}(1)_{V} .
$$

This model can support left-right discrete symmetry as well as $\mathrm{q}-\ell$ symmetry [10]. An even simpler extension sees only the $\mathrm{U}(1)$ subgroup of $\mathrm{SU}(2)_{R}$ gauged. Any

gauge extension like this will also require an extended Higgs sector. We will not probe this issue any further in this paper, but have mentioned it here for the sake of completeness.

\section{Study of a realistic Higgs sector}


We now study the two models using Higgs sector B in more detail. We will address the following issues: (i) the construction and minimization of the Higgs potentials, (ii) the spectrum and Yukawa couplings of the physical Higgs bosons, and (iii) the phenomenological bounds obtained from tree-level flavour-changing neutral Higgs boson effects in the light $\phi_{1,2}$ sector. Models 1 and 2 differ from each other in important ways, and so we will examine them separately.

\subsection{Model 1}

The first issue is the minimization of the Higgs potential: we have to check that the desired symmetry breaking pattern,

$$
\left\langle\phi_{1}\right\rangle=\left(\begin{array}{c}
0 \\
u_{1}
\end{array}\right), \quad\left\langle\phi_{2}\right\rangle=\left(\begin{array}{c}
u_{2} e^{i \xi} \\
0
\end{array}\right), \quad\left\langle\chi_{1}\right\rangle=0, \quad\left\langle\chi_{2}\right\rangle=\left(\begin{array}{l}
v \\
0 \\
0
\end{array}\right)
$$

is possible. In this equation we have chosen $u_{1}, u_{2}, v>0$ by a phase convention, and we have also taken the possible phase angle $\xi$ to reside with $\phi_{2}$.

In order to construct the Higgs potential, we first write down all gauge-invariant terms with definite transformation properties under $\mathrm{q}-\ell$ symmetry that are quadratic in the Higgs boson fields. The terms $E$ that are even $(E \rightarrow E)$ under the discrete symmetry are,

$$
E_{1} \equiv \phi_{1}^{\dagger} \phi_{1}+\phi_{2}^{\dagger} \phi_{2} \quad \text { and } \quad E_{2} \equiv \chi_{1}^{\dagger} \chi_{1}+\chi_{2}^{\dagger} \chi_{2}
$$

The terms $O$ that are odd $(O \rightarrow-O)$ are,

$$
O_{1} \equiv \phi_{1}^{\dagger} \phi_{1}-\phi_{2}^{\dagger} \phi_{2}, \quad O_{2} \equiv \chi_{1}^{\dagger} \chi_{1}-\chi_{2}^{\dagger} \chi_{2}, \quad O_{3} \equiv \phi_{1} \phi_{2}, \quad \text { and } \quad O_{3}^{\dagger} \equiv\left(\phi_{1} \phi_{2}\right)^{\dagger} \text {, }
$$

where

$$
\phi_{1} \phi_{2} \equiv \phi_{1}^{\top} i \tau_{2} \phi_{2}=\phi_{1}^{+} \phi_{2}^{-}-\phi_{1}^{0} \phi_{2}^{0}
$$

The Higgs potential $V$ is obtained from these terms as follows:

$$
\begin{aligned}
V & =\mu_{\phi}^{2} E_{1}+\mu_{\chi}^{2} E_{2}+\lambda_{1} E_{1}^{2}+\lambda_{2} E_{2}^{2} \\
& +\lambda_{3} O_{1}^{2}+\lambda_{4} O_{2}^{2}+\left[\lambda_{5} O_{3}^{2}+\text { H.c. }\right] \\
& +\lambda_{6} E_{1} E_{2}+\lambda_{7} O_{1} O_{2}+\left[\lambda_{8} O_{1} O_{3}+\text { H.c. }\right] \\
& +\left[\lambda_{9} O_{2} O_{3}+\text { H.c. }\right]+\lambda_{10} O_{3} O_{3}^{\dagger}
\end{aligned}
$$

where $\mu_{\phi, \chi}^{2}, \lambda_{1-4,6,7,10}$ are real numbers and $\lambda_{5,8,9}$ are in general complex. There are no quartic terms that cannot be written down as the product of $E$ s and $O$ s. 
The minimization equations for the Higgs potential written in this form are not particularly enlightening, and it is difficult to tell if minima are local or global. The terms $\mu_{\phi, \chi}^{2}$ and $\lambda_{1-4,6}$ can actually be written in a much more useful form. We will call the resulting partial Higgs potential $V_{\text {large }}$, and it is given by

$$
\begin{aligned}
V_{\text {large }} & =\lambda_{1}^{\prime}\left(\phi_{1}^{\dagger} \phi_{1}+\phi_{2}^{\dagger} \phi_{2}-u_{1}^{2}-u_{2}^{2}\right)^{2}+\lambda_{2}^{\prime}\left(\chi_{1}^{\dagger} \chi_{1}+\chi_{2}^{\dagger} \chi_{2}-v^{2}\right)^{2} \\
& +\lambda_{6}^{\prime}\left(\phi_{1}^{\dagger} \phi_{1}+\phi_{2}^{\dagger} \phi_{2}+\chi_{1}^{\dagger} \chi_{1}+\chi_{2}^{\dagger} \chi_{2}-u_{1}^{2}-u_{2}^{2}-v^{2}\right)^{2} \\
& +\lambda_{4}^{\prime}\left(\chi_{1}^{\dagger} \chi_{1}\right)\left(\chi_{2}^{\dagger} \chi_{2}\right)+\lambda_{3}^{\prime}\left[\left(\phi_{1}^{\dagger} \phi_{1}\right)\left(\phi_{2}^{\dagger} \phi_{2}\right)-\left(\phi_{1} \phi_{2}\right)\left(\phi_{1} \phi_{2}\right)^{\dagger}\right] .
\end{aligned}
$$

The remaining terms in the full Higgs potential are assembled into a contribution called $V_{\text {small }}$ so that $V=V_{\text {large }}+V_{\text {small }}$.

Consider the parameter space region given by $\lambda_{1-4,6}^{\prime}>0$. The first three terms in $V_{\text {large }}$ are clearly minimized by taking $\left\langle\phi_{1}^{\dagger} \phi_{1}\right\rangle+\left\langle\phi_{2}^{\dagger} \phi_{2}\right\rangle=u_{1}^{2}+u_{2}^{2}$ and $\left\langle\chi_{1}^{\dagger} \chi_{1}\right\rangle+\left\langle\chi_{2}^{\dagger} \chi_{2}\right\rangle=$ $v^{2}$. The fourth term is minimized if either $\left\langle\chi_{1}\right\rangle=0$ or $\left\langle\chi_{2}\right\rangle=0$. Without loss of generality we may take $\left\langle\chi_{1}\right\rangle=0$, thereby being consistent with our previous exposition. The fourth term thus guarantees leptonic colour breaking, $q-\ell$ symmetry breaking and the preservation of quark colour as an exact symmetry. The last term guarantees that electromagnetic gauge invariance remains exact. The argument goes like this: We start by using a weak-isospin rotation to transform to the basis where $\left\langle\phi_{1}^{ \pm}\right\rangle=0$. This however does not ensure that $\left\langle\phi_{2}^{ \pm}\right\rangle=0$. But the last term in Eq. (31) at the minimum is then just $\lambda_{3}^{\prime}\left\langle\phi_{2}^{-} \phi_{2}^{+}\right\rangle\left|\left\langle\phi_{1}^{0}\right\rangle\right|^{2}$. Since $\lambda_{3}^{\prime}>0$, then either $\left\langle\phi_{2}^{ \pm}\right\rangle=0$ or $\left\langle\phi_{1}^{0}\right\rangle=0$, and so we choose the former.

We have thus shown that the VEV pattern of Eq. (26) arises when all the $\lambda^{\prime}$ coupling constants in $V_{\text {large }}$ are positive, provided that the omitted terms in $V_{\text {small }}$ are small enough. We have not shown that this is the only region of parameter space that will do, only that it is an example of a suitable region. This is perhaps fortunate, because this region has a serious drawback: the existence of a light pseudo-Goldstone boson. The point is that $V_{\text {large }}$ is invariant under independent phase rotations for $\chi_{2}$, $\phi_{1}$ and $\phi_{2}$ that are all spontaneously broken. Two of the resulting Goldstone bosons are eaten, but one remains as a light physical boson. It will pick up some mass from $V_{\text {small }}$ and via radiative corrections from the Yukawa Lagrangian, but the fear is that it will be light enough to mediate unacceptably large neutral flavour-changing processes.

This problem has arisen because the $\phi_{1} \phi_{2}$ combination is odd under the discrete symmetry, and thus cannot appear in Higgs potential terms of the form (positive number $) \times\left(\phi_{1} \phi_{2}+u_{1} u_{2} \cos \xi\right)^{2}$. Terms like $\left(\phi_{1} \phi_{2}\right)^{2}$ eliminate the spurious phase invariance, but they seemingly cannot be written in a manifestly useful way for purposes of easy minimization while at the same time respecting the discrete symmetry. 
However, we can easily convince ourselves that a pseudo-Goldstone boson does not necessarily always accompany our required VEV pattern. Let us write down an effective Higgs potential for $\phi_{1}$ and $\phi_{2}$ after a nonzero VEV for $\chi_{2}$ has already spontaneously broken the discrete symmetry. We do this by allowing the soft discrete symmetry breaking quadratic terms $\phi_{1} \phi_{2}$ and $\phi_{1}^{\dagger} \phi_{1}-\phi_{2}^{\dagger} \phi_{2}$ to appear in our effective potential. The most general form is

$$
\begin{aligned}
V_{\mathrm{eff}} & =\lambda_{1}^{\prime \prime}\left(\phi_{1}^{\dagger} \phi_{1}+\phi_{2}^{\dagger} \phi_{2}-u_{1}^{2}-u_{2}^{2}\right)^{2} \\
& +\lambda_{3}^{\prime \prime}\left[\left(\phi_{1}^{\dagger} \phi_{1}\right)\left(\phi_{2}^{\dagger} \phi_{2}\right)-\left(\phi_{1} \phi_{2}\right)\left(\phi_{1} \phi_{2}\right)^{\dagger}\right]+\lambda_{7}^{\prime \prime}\left(\phi_{1}^{\dagger} \phi_{1}-\phi_{2}^{\dagger} \phi_{2}-u_{1}^{2}+u_{2}^{2}\right)^{2} \\
& +\lambda_{5}^{\prime \prime}\left[\left(\phi_{1} \phi_{2}\right)+\left(\phi_{1} \phi_{2}\right)^{\dagger}+2 u_{1} u_{2} \cos \xi\right]^{2} \\
& +\tilde{\lambda}_{5}^{\prime \prime}\left[i\left(\phi_{1} \phi_{2}\right)-i\left(\phi_{1} \phi_{2}\right)^{\dagger}-2 u_{1} u_{2} \sin \xi\right]^{2} \\
& +\lambda_{8}^{\prime \prime}\left[\phi_{1}^{\dagger} \phi_{1}-\phi_{2}^{\dagger} \phi_{2}+\left(\phi_{1} \phi_{2}\right)+\left(\phi_{1} \phi_{2}\right)^{\dagger}-u_{1}^{2}+u_{2}^{2}+2 u_{1} u_{2} \cos \xi\right]^{2} \\
& +\tilde{\lambda}_{8}^{\prime \prime}\left[\phi_{1}^{\dagger} \phi_{1}-\phi_{2}^{\dagger} \phi_{2}+i\left(\phi_{1} \phi_{2}\right)-i\left(\phi_{1} \phi_{2}\right)^{\dagger}-u_{1}^{2}+u_{2}^{2}-2 u_{1} u_{2} \sin \xi\right]^{2} \\
& +\lambda_{10}^{\prime \prime}\left[\left(\phi_{1} \phi_{2}\right)+\left(\phi_{1} \phi_{2}\right)^{\dagger}\right. \\
& \left.\quad+i\left(\phi_{1} \phi_{2}\right)-i\left(\phi_{1} \phi_{2}\right)^{\dagger}+2 u_{1} u_{2} \cos \xi-2 u_{1} u_{2} \sin \xi\right]^{2} .
\end{aligned}
$$

If all the $\lambda^{\prime \prime}$ parameters are positive, then the required pattern of symmetry breaking follows. Furthermore, there is no reason to make the terms breaking the spurious phase invariance small, and so the putative pseudo-Goldstone boson is eliminated. This result shows that some of the terms in $V_{\text {small }}$, which are putatively small, can actually be large enough to solve this pseudo-problem without inducing the spontaneous breaking of electromagnetic gauge invariance, or otherwise spoiling our desired symmetry breaking pattern.

We now exhibit the physical Higgs fields. Writing

$$
\phi_{1}^{0}=u_{1}+\frac{h_{1}+i \eta_{1}}{\sqrt{2}}, \quad \phi_{2}^{0}=u_{2}+\frac{h_{2}+i \eta_{2}}{\sqrt{2}}, \quad \chi_{2}^{0}=v+\frac{H+i E}{\sqrt{2}},
$$

where for simplicity we have put the CP-violating phase $\xi$ to zero, we identify the unphysical neutral Goldstone boson fields as

$$
g^{0}=\frac{u_{2} \eta_{2}-u_{1} \eta_{1}}{\sqrt{u_{1}^{2}+u_{2}^{2}}} \text { and } E,
$$

where $g^{0}$ is the field eaten by the $Z^{0}$. The field $E$ is eaten by a $Z^{\prime}$ boson arising from the spontaneous breakdown of leptonic colour. The field we called $\tilde{\chi}_{2}$ in Eq. (8) is also eaten when leptonic colour breaks. The unphysical charged Goldstone bosons are

$$
g^{ \pm} \equiv \frac{u_{2} \phi_{2}^{ \pm}-u_{1} \phi_{1}^{ \pm}}{\sqrt{u_{1}^{2}+u_{2}^{2}}}
$$


and they are of course eaten by $W^{ \pm}$. From now on we will work in unitary gauge, so that these unphysical fields will simply be set to zero in the Yukawa Lagrangians.

The physical Higgs fields consist of the charged field $H^{ \pm}$orthogonal to $g^{ \pm}$, where

$$
H^{ \pm} \equiv \frac{u_{2} \phi_{1}^{ \pm}+u_{1} \phi_{2}^{ \pm}}{\sqrt{u_{1}^{2}+u_{2}^{2}}}
$$

the CP-odd field $\eta$ orthogonal to $g^{0}$, where

$$
\eta \equiv \frac{u_{2} \eta_{1}+u_{1} \eta_{2}}{\sqrt{u_{1}^{2}+u_{2}^{2}}}
$$

and three CP-even fields whose mass eigenstates are linear combinations of $h_{1}, h_{2}$ and $H$. Now, in most of parameter space the field $H$ mixes very little with the fields $h_{1,2}$, because the scale of leptonic colour breaking has to be significantly higher than the electroweak scale. We will concentrate on this large region of parameter space in the rest of this paper. We therefore approximately write the mass eigenstate fields as $h_{1,2}^{\prime}$ and $H$, where

$$
\left(\begin{array}{l}
h_{1}^{\prime} \\
h_{2}^{\prime}
\end{array}\right)=\left(\begin{array}{cc}
\cos \phi & \sin \phi \\
-\sin \phi & \cos \phi
\end{array}\right)\left(\begin{array}{l}
h_{1} \\
h_{2}
\end{array}\right),
$$

for some mixing angle $\phi$. We can of course relate $\phi$ to the parameters in the Higgs potential, but we will not need to know this expression.

The most useful way to write the Yukawa Lagrangian is to replace the $\Lambda$ parameters in Eq. (17) by the mass matrices through Eq. (18). We then obtain that

$$
\begin{aligned}
\mathcal{L}_{\mathrm{Yuk}} & =\frac{1}{u} \bar{Q}_{L}\left(m_{u} \Phi_{1}+m_{e} \Phi_{2}\right) u_{R}+\frac{1}{u} \bar{F}_{L}\left(m_{u} \Phi_{2}^{c}-m_{e} \Phi_{1}^{c}\right) E_{R} \\
& +\frac{1}{u} \bar{Q}_{L}\left(m_{\nu}^{\text {Dirac }} \Phi_{2}^{c}-m_{d} \Phi_{1}^{c}\right) d_{R}+\frac{1}{u} \bar{F}_{L}\left(m_{\nu}^{\text {Dirac }} \Phi_{1}+m_{d} \Phi_{2}\right) N_{R}+\text { H.c. }
\end{aligned}
$$

where $u \equiv \sqrt{u_{1}^{2}+u_{2}^{2}}$ and

$$
\begin{aligned}
\Phi_{1} & \equiv \frac{u_{2} \phi_{2}+u_{1} \phi_{1}^{c}}{u}=\left(\begin{array}{c}
u+\frac{u_{1} h_{1}+u_{2} h_{2}}{\sqrt{2} u}+i \frac{g^{0}}{\sqrt{2}} \\
g^{-}
\end{array}\right), \\
\Phi_{2} & \equiv \frac{u_{1} \phi_{2}-u_{2} \phi_{1}^{c}}{u}=\left(\begin{array}{c}
\frac{u_{1} h_{2}-u_{2} h_{1}}{\sqrt{2} u}+i \frac{\eta}{\sqrt{2}} \\
H^{-}
\end{array}\right),
\end{aligned}
$$


and $\Phi_{1,2}^{c} \equiv i \tau_{2} \Phi_{1,2}^{*}$. Under q- $\ell$ symmetry,

$$
\Phi_{1} \leftrightarrow \Phi_{2}^{c} \quad \text { and } \quad \Phi_{2} \leftrightarrow-\Phi_{1}^{c}
$$

and the electroweak symmetry breaking VEVs are $\left\langle\Phi_{1}\right\rangle=u$ and $\left\langle\Phi_{2}\right\rangle=0$.

By using Eq. (41), the Yukawa Lagrangians involving the physical mass eigenstate fields $H^{ \pm}$and $\eta$ can be easily read off Eq. (39). They are,

$$
\begin{aligned}
\mathcal{L}_{\text {Yuk }}^{+} & =\frac{1}{u} \bar{d}_{L} m_{E} u_{R} H^{-}+\frac{1}{u} \bar{u}_{L} m_{\nu}^{\text {Dirac }} d_{R} H^{+} \\
& +\frac{1}{u} \bar{N}_{L} m_{u} E_{R} H^{+}+\frac{1}{u} \bar{E}_{L} m_{d} N_{R} H^{-}+\text {H.c. }
\end{aligned}
$$

and

$$
\begin{aligned}
\mathcal{L}_{\text {Yuk }}^{\eta} & =\frac{i}{\sqrt{2} u} \bar{u}_{L} m_{E} u_{R} \eta+\frac{i}{\sqrt{2} u} \bar{E}_{L} m_{u} E_{R} \eta \\
& +\frac{i}{\sqrt{2} u} \bar{d}_{L} m_{\nu}^{\text {Dirac }} d_{R} \eta+\frac{i}{\sqrt{2} u} \bar{N}_{L} m_{d} N_{R} \eta+\text { H.c. }
\end{aligned}
$$

These Yukawa Lagrangians are extremely interesting, because the discrete $q-\ell$ symmetry is seen to act in a highly non-trivial way: The Yukawa coupling constants for quarks (leptons) are proportional to the mass matrices of the corresponding discrete symmetry partner leptons (quarks). This is a rather different situation from the usual expectation that the Yukawa coupling constants for fermion $f$ should be proportional to the mass $m_{f}$ of that same fermion. Note in particular that the downquark flavour-changing neutral couplings of the $\eta$ Higgs boson are proportional to the Dirac masses of the neutrinos. Since neutrino Dirac masses are constrained to be very small, we see that neutral flavour changing processes mediated by $\eta$ are highly suppressed for down quarks. This means, most importantly, that no useful bound is obtained from $K-\bar{K}$ mixing on the tree-level flavour-changing $\eta$ couplings. The reader should also note that the fermion fields in Eqs. (43) and (44) are weak-interaction eigenstates, not mass eigenstates. In the mass eigenstate basis, two unitary diagonalization matrices would also appear in the Lagrangians, a point we will return to later on.

\footnotetext{
${ }^{7}$ If we extend the Higgs sector to accomodate the see-saw mechanism (see Higgs sector C above) then of course this qualitative conclusion no longer holds, because neutrino Dirac masses can then be large. We also point out that in the Higgs sector B scenario we have no explanation for why the neutrino Dirac masses should be so small. Our result is simply that given tiny neutrino Dirac masses, then tiny down-quark sector flavour-changing neutral couplings follow.
} 
Using the definition

$$
\tan \omega \equiv u_{2} / u_{1}
$$

we can write the interaction Lagrangian of the fermions with the mass eigenstate CP-even bosons $h_{1,2}^{\prime}$ as

$$
\begin{aligned}
& \mathcal{L}_{\text {Yuk }}^{h}=\frac{1}{\sqrt{2} u} \bar{u}_{L}\left[m_{u}\left\{\cos (\omega-\phi) h_{1}^{\prime}+\sin (\omega-\phi) h_{2}^{\prime}\right\}\right. \\
& \left.+m_{e}\left\{-\sin (\omega-\phi) h_{1}^{\prime}+\cos (\omega-\phi) h_{2}^{\prime}\right\}\right] u_{R} \\
& +\frac{1}{\sqrt{2} u} \bar{E}_{L}\left[m_{u}\left\{\sin (\omega-\phi) h_{1}^{\prime}-\cos (\omega-\phi) h_{2}^{\prime}\right\}\right. \\
& \left.+m_{e}\left\{\cos (\omega-\phi) h_{1}^{\prime}+\sin (\omega-\phi) h_{2}^{\prime}\right\}\right] E_{R} \\
& +\frac{1}{\sqrt{2} u} \bar{d}_{L}\left[m_{d}\left\{\cos (\omega-\phi) h_{1}^{\prime}+\sin (\omega-\phi) h_{2}^{\prime}\right\}\right. \\
& \left.+m_{\nu}^{\text {Dirac }}\left\{\sin (\omega-\phi) h_{1}^{\prime}-\cos (\omega-\phi) h_{2}^{\prime}\right\}\right] d_{R} \\
& +\frac{1}{\sqrt{2} u} \bar{N}_{L}\left[m_{d}\left\{-\sin (\omega-\phi) h_{1}^{\prime}+\cos (\omega-\phi) h_{2}^{\prime}\right\}\right. \\
& \left.+m_{\nu}^{\text {Dirac }}\left\{\cos (\omega-\phi) h_{1}^{\prime}+\sin (\omega-\phi) h_{2}^{\prime}\right\}\right] N_{R}+\text { H.c. }
\end{aligned}
$$

where again interaction eigenstates have been used for the fermions. This Lagrangian is also very interesting, because in the fermion mass eigenstate basis it is clear that the flavour-changing contributions for a given fermion are always proportional to the mass matrix of its $q-\ell$ partner (multiplied by diagonalization matrices). This result is similar to that obtained for the boson $\eta$. Once again, the most important consequence of this is the large suppression of down-quark sector flavour-changing neutral Higgs effects because of their proportionality to tiny neutrino Dirac mass matrices. Note, however, that if we set $m_{\nu}^{\text {Dirac }}=0$ exactly, then all interactions between $\eta$ and the down-quarks disappear, whereas the $h_{1,2}$ fields still have interactions although they are now strictly flavour-diagonal.

Having made the important discovery that discrete $q-\ell$ symmetry plays an interesting and important role in constraining the Yukawa interactions of physical Higgs bosons and fermions in Model 1, we now turn to a similar analysis of Model 2. We will then return to Model 1 when we come to examine phenomenological bounds on tree-level flavour-changing neutral processes in a subsequent subsection.

\subsection{Model 2}


Happily, the analysis of the Higgs potential for Model 2 is much simpler than for Model 1. This is because the combination $\phi_{1} \phi_{2} \equiv \phi_{1}^{\top} i \tau_{2} \phi_{2}$ is now even under the discrete $\mathrm{q}-\ell$ symmetry. The even and odd quadratic combinations are now given by

$$
E_{1} \equiv \phi_{1}^{\dagger} \phi_{1}+\phi_{2}^{\dagger} \phi_{2}, \quad E_{2} \equiv \chi_{1}^{\dagger} \chi_{1}+\chi_{2}^{\dagger} \chi_{2}, \quad E_{3} \equiv \phi_{1} \phi_{2}, \quad E_{3}^{\dagger} \equiv\left(\phi_{1} \phi_{2}\right)^{\dagger}
$$

and

$$
O_{1} \equiv \phi_{1}^{\dagger} \phi_{1}-\phi_{2}^{\dagger} \phi_{2}, \quad O_{2} \equiv \chi_{1}^{\dagger} \chi_{1}-\chi_{2}^{\dagger} \chi_{2}
$$

respectively. As for Model 1, the Higgs potential $V$ can be written as sums of $E, E^{2}$ and $O^{2}$ forms. However, this time we can write all but one of these terms immediately in a form useful for answering minimization questions. The result is

$$
\begin{aligned}
V_{\text {large }} & =\lambda_{1}^{\prime}\left(\phi_{1}^{\dagger} \phi_{1}+\phi_{2}^{\dagger} \phi_{2}-u_{1}^{2}-u_{2}^{2}\right)^{2}+\lambda_{2}^{\prime}\left(\chi_{1}^{\dagger} \chi_{1}+\chi_{2}^{\dagger} \chi_{2}-v^{2}\right)^{2} \\
& +\lambda_{3}^{\prime}\left[\left(\phi_{1}^{\dagger} \phi_{1}\right)\left(\phi_{2}^{\dagger} \phi_{2}\right)-\left(\phi_{1} \phi_{2}\right)\left(\phi_{1} \phi_{2}\right)^{\dagger}\right]+\lambda_{4}^{\prime}\left(\chi_{1}^{\dagger} \chi_{1}\right)\left(\chi_{2}^{\dagger} \chi_{2}\right) \\
& +\lambda_{5}^{\prime}\left[\phi_{1} \phi_{2}+\left(\phi_{1} \phi_{2}\right)^{\dagger}+2 u_{1} u_{2} \cos \xi\right]^{2} \\
& +\tilde{\lambda}_{5}^{\prime}\left[i \phi_{1} \phi_{2}-i\left(\phi_{1} \phi_{2}\right)^{\dagger}-2 u_{1} u_{2} \sin \xi\right]^{2} \\
& +\lambda_{6}^{\prime}\left(\phi_{1}^{\dagger} \phi_{1}+\phi_{2}^{\dagger} \phi_{2}+\chi_{1}^{\dagger} \chi_{1}+\chi_{2}^{\dagger} \chi_{2}-u_{1}^{2}-u_{2}^{2}-v^{2}\right)^{2} \\
& +\lambda_{8}^{\prime}\left[\phi_{1}^{\dagger} \phi_{1}+\phi_{2}^{\dagger} \phi_{2}+\phi_{1} \phi_{2}+\left(\phi_{1} \phi_{2}\right)^{\dagger}-u_{1}^{2}-u_{2}^{2}+2 u_{1} u_{2} \cos \xi\right]^{2} \\
& +\tilde{\lambda}_{8}^{\prime}\left[\phi_{1}^{\dagger} \phi_{1}+\phi_{2}^{\dagger} \phi_{2}+i \phi_{1} \phi_{2}-i\left(\phi_{1} \phi_{2}\right)^{\dagger}-u_{1}^{2}-u_{2}^{2}-2 u_{1} u_{2} \sin \xi\right]^{2} \\
& +\lambda_{9}^{\prime}\left[\chi_{1}^{\dagger} \chi_{1}+\chi_{2}^{\dagger} \chi_{2}+\phi_{1} \phi_{2}+\left(\phi_{1} \phi_{2}\right)^{\dagger}+2 u_{1} u_{2} \cos \xi-v^{2}\right]^{2} \\
& +\tilde{\lambda}_{9}^{\prime}\left[\chi_{1}^{\dagger} \chi_{1}+\chi_{2}^{\dagger} \chi_{2}+i \phi_{1} \phi_{2}-i\left(\phi_{1} \phi_{2}\right)^{\dagger}-2 u_{1} u_{2} \sin \xi-v^{2}\right]^{2} \\
& +\lambda_{10}^{\prime}\left[\phi_{1} \phi_{2}+\left(\phi_{1} \phi_{2}\right)^{\dagger}\right. \\
& \left.\quad+i \phi_{1} \phi_{2}-i\left(\phi_{1} \phi_{2}\right)^{\dagger}+2 u_{1} u_{2} \cos \xi-2 u_{1} u_{2} \sin \xi\right]^{2} .
\end{aligned}
$$

If we take all the $\lambda^{\prime}$ parameters above to be positive, then the correct symmetry breaking pattern is assured, provided that the one omitted term, namely $V_{7}$ where

$$
V_{7}=\lambda_{7}\left(\phi_{1}^{\dagger} \phi_{1}-\phi_{2}^{\dagger} \phi_{2}\right)\left(\chi_{1}^{\dagger} \chi_{1}-\chi_{2}^{\dagger} \chi_{2}\right)
$$

is small enough, or innocuous enough. If we want to we can partially incorporate this term in our analysis by writing an effective soft-breaking term of the form $\lambda_{7}^{\prime \prime}\left[O_{1}-u_{1}^{2}+u_{2}^{2}\right]^{2}$ in an effective potential after leptonic colour breakdown, but there is no practical need to examine this term more closely.

The identification of physical and unphysical fields is exactly the same as for Model 1. The Yukawa Lagrangians are, however, a little different. The charged Higgs boson Yukawa Lagrangian is

$$
\mathcal{L}_{\text {Yuk }}^{+}=\frac{1}{u\left(u_{2}^{2}-u_{1}^{2}\right)} \bar{d}_{L}\left(2 u_{1} u_{2} m_{u}-u^{2} m_{e}\right) u_{R} H^{-}
$$




$$
\begin{aligned}
& +\frac{1}{u\left(u_{2}^{2}-u_{1}^{2}\right)} \bar{N}_{L}\left(u^{2} m_{u}-2 u_{1} u_{2} m_{e}\right) E_{R} H^{+} \\
& +\frac{1}{u\left(u_{2}^{2}-u_{1}^{2}\right)} \bar{u}_{L}\left(-2 u_{1} u_{2} m_{d}+u^{2} m_{\nu}^{\text {Dirac }}\right) d_{R} H^{+} \\
& +\frac{1}{u\left(u_{2}^{2}-u_{1}^{2}\right)} \bar{E}_{L}\left(-u^{2} m_{d}+2 u_{1} u_{2} m_{\nu}^{\text {Dirac }}\right) N_{R} H^{-}+\text {H.c. }
\end{aligned}
$$

Note that the $\mathrm{q}-\ell$ partnership is manifested in a more complicated way for this Lagrangian compared with its analogue in Model 1.

The CP-odd neutral particle $\eta$ enjoys the following interactions:

$$
\begin{aligned}
\mathcal{L}_{\text {Yuk }}^{\eta} & =\frac{i}{\sqrt{2} u\left(u_{2}^{2}-u_{1}^{2}\right)} \bar{u}_{L}\left(2 u_{1} u_{2} m_{u}-u^{2} m_{e}\right) u_{R} \eta \\
& +\frac{i}{\sqrt{2} u\left(u_{2}^{2}-u_{1}^{2}\right)} \bar{E}_{L}\left(u^{2} m_{u}-2 u_{1} u_{2} m_{e}\right) E_{R} \eta \\
& +\frac{i}{\sqrt{2} u\left(u_{2}^{2}-u_{1}^{2}\right)} \bar{d}_{L}\left(-2 u_{1} u_{2} m_{d}+u^{2} m_{\nu}^{\text {Dirac }}\right) d_{R} \eta \\
& +\frac{i}{\sqrt{2} u\left(u_{2}^{2}-u_{1}^{2}\right)} \bar{N}_{L}\left(-u^{2} m_{u}+2 u_{1} u_{2} m_{e}\right) N_{R} \eta+\text { H.c. }
\end{aligned}
$$

As for Model 1, the flavour-changing interaction of $\eta$ for a given fermion class is proportional to the mass matrix of the discrete symmetry partner of that fermion. Most importantly, the down-quark neutral flavour-violating piece is once again proportional to the Dirac mass matrix of the neutrino and is therefore very small. A difference from Model 1 is that there is non-zero piece for a given fermion proportional to the mass matrix of that same fermion (and is thus diagonal in the mass eigenstate basis).

The CP-even mass eigenstate Higgs bosons $h_{1,2}^{\prime}$ have an interaction Lagrangian given by

$$
\begin{aligned}
\mathcal{L}_{\text {Yuk }}^{h}= & \frac{u}{\sqrt{2}\left(u_{2}^{2}-u_{1}^{2}\right)} \bar{u}_{L}\left[m_{u}\left\{-\cos (\omega+\phi) h_{1}^{\prime}+\sin (\omega+\phi) h_{2}^{\prime}\right\}\right. \\
+ & \frac{\left.u m_{e}\left\{\sin (\omega-\phi) h_{1}^{\prime}-\cos (\omega-\phi) h_{2}^{\prime}\right\}\right] u_{R}}{\sqrt{2}\left(u_{2}^{2}-u_{1}^{2}\right)} \bar{E}_{L}\left[m_{u}\left\{\sin (\omega-\phi) h_{1}^{\prime}-\cos (\omega-\phi) h_{2}^{\prime}\right\}\right. \\
& \left.\quad+m_{e}\left\{-\cos (\omega+\phi) h_{1}^{\prime}+\sin (\omega+\phi) h_{2}^{\prime}\right\}\right] E_{R} \\
+ & \frac{u}{\sqrt{2}\left(u_{2}^{2}-u_{1}^{2}\right)} \bar{d}_{L}\left[m_{d}\left\{-\cos (\omega+\phi) h_{1}^{\prime}+\sin (\omega+\phi) h_{2}^{\prime}\right\}\right.
\end{aligned}
$$




$$
\begin{gathered}
\left.+m_{\nu}^{\text {Dirac }}\left\{\sin (\omega-\phi) h_{1}^{\prime}-\cos (\omega-\phi) h_{2}^{\prime}\right\}\right] d_{R} \\
+\frac{u}{\sqrt{2}\left(u_{2}^{2}-u_{1}^{2}\right)} \bar{N}_{L}\left[m_{d}\left\{\sin (\omega-\phi) h_{1}^{\prime}-\cos (\omega-\phi) h_{2}^{\prime}\right\}\right. \\
\left.+m_{\nu}^{\text {Dirac }}\left\{-\cos (\omega+\phi) h_{1}^{\prime}+\sin (\omega+\phi) h_{2}^{\prime}\right\}\right] N_{R}+\text { H.c. }
\end{gathered}
$$

As the reader can easily see, the flavour-changing interaction for a given fermion class is proportional to the mass matrix of its $q-\ell$ symmetry partner. Once again, down-quark sector neutral flavour-violating processes are zero if the neutrino Dirac masses are zero.

\subsection{Phenomenology}

In this section we will present an overview of the phenomenological implications of Models 1 and 2. The main interest is on the tree-level neutral flavour-changing effects mediated by $\eta, h_{1}^{\prime}$ and $h_{2}^{\prime}$. (We will not cover loop effects quantitatively in this paper; we will be content to qualitatively discuss the most interesting of these here. We hope to return to a more detailed phenomenological analysis in future work.)

\subsubsection{Tree-level neutral flavour-changing effects.}

Let the mass eigenstate fermion field $f$ be denoted by $f^{\prime}$. We introduce the leftand right-sector unitary diagonalization matrices $V_{L, R}^{f}$ through,

$$
f_{L}^{\prime} \equiv V_{L}^{f} f_{L}, \quad \text { and } \quad f_{R}^{\prime} \equiv V_{R}^{f} f_{R}
$$

where $f=u, d, e, \nu$. The corresponding diagonal mass matrices are given by

$$
m_{f}^{\text {diag }}=V_{L}^{f} m_{f} V_{R}^{f \dagger} .
$$

We now rewrite the flavour-changing pieces of the neutral Higgs Yukawa Lagrangians in terms of mass eigenstate fermions.

For Model 1 we obtain,

$$
\begin{aligned}
\mathcal{L}_{\mathrm{FC}}^{\eta} & =\frac{1}{\sqrt{2} u} \bar{u}_{L}^{\prime} V_{L}^{u} V_{L}^{e \dagger} m_{e}^{\text {diag }} V_{R}^{e} V_{R}^{u \dagger} u_{R}^{\prime} \eta+\frac{1}{\sqrt{2} u} \bar{e}_{L}^{\prime} V_{L}^{e} V_{L}^{u \dagger} m_{u}^{\text {diag }} V_{R}^{u} V_{R}^{e \dagger} e_{R}^{\prime} \eta \\
& +\frac{1}{\sqrt{2} u} \bar{d}_{L}^{\prime} V_{L}^{d} V_{L}^{\nu \dagger} m_{\nu}^{\text {diag }} V_{R}^{\nu} V_{R}^{d \dagger} d_{R}^{\prime} \eta+\frac{1}{\sqrt{2} u} \bar{\nu}_{L}^{\prime} V_{L}^{\nu} V_{L}^{d \dagger} m_{d}^{\text {diag }} V_{R}^{d} V_{R}^{\nu \dagger} \nu_{R}^{\prime} \eta ;
\end{aligned}
$$


together with

$$
\begin{aligned}
\mathcal{L}_{\mathrm{FC}}^{h} & =\frac{1}{\sqrt{2} u} \bar{u}_{L}^{\prime} V_{L}^{u} V_{L}^{e \dagger} m_{e}^{\mathrm{diag}} V_{R}^{e} V_{R}^{u \dagger} u_{R}^{\prime}\left[\sin (\omega-\phi) h_{1}^{\prime}+\cos (\omega-\phi) h_{2}^{\prime}\right] \\
& +\frac{1}{\sqrt{2} u} \bar{e}_{L}^{\prime} V_{L}^{e} V_{L}^{u \dagger} m_{u}^{\mathrm{diag}} V_{R}^{u} V_{R}^{e \dagger} e_{R}^{\prime}\left[\sin (\omega-\phi) h_{1}^{\prime}-\cos (\omega-\phi) h_{2}^{\prime}\right] \\
& +\frac{1}{\sqrt{2} u} \bar{d}_{L}^{\prime} V_{L}^{d} V_{L}^{\nu \dagger} m_{\nu}^{\operatorname{diag}} V_{R}^{\nu} V_{R}^{d \dagger} d_{R}^{\prime}\left[\sin (\omega-\phi) h_{1}^{\prime}-\cos (\omega-\phi) h_{2}^{\prime}\right] \\
& +\frac{1}{\sqrt{2} u} \bar{\nu}_{L}^{\prime} V_{L}^{\nu} V_{L}^{d \dagger} m_{d}^{\operatorname{diag}} V_{R}^{d} V_{R}^{\nu \dagger} \nu_{R}^{\prime}\left[-\sin (\omega-\phi) h_{1}^{\prime}+\cos (\omega-\phi) h_{2}^{\prime}\right],
\end{aligned}
$$

for flavour-changing $\eta$ and $h_{1,2}^{\prime}$ interactions respectively. (Note that the two Lagrangians above also contain flavour-diagonal terms.) The corresponding Lagrangians for Model 2 are easily discerned from Eqs. (52) and (53). They can be obtained from the two Model 1 Lagrangians above by making the substitution $u \rightarrow\left(u_{2}^{2}-u_{1}^{2}\right) / u$ and by changing some of the plus and minus signs.

Let us make some qualitative observations: (i) As we have emphasized, if the neutrino Dirac masses are zero, then there are no tree-level down-quark sector interactions which change flavour. In this case, there are also no neutrino-sector flavourchanging vertices, because we are free to redefine the neutrino fields by use of the down-sector diagonalization matrices. (ii) All the action is therefore in the up-quark and charged-lepton sectors. Since the up-quark sector masses are larger than corresponding charged-lepton masses, the largest flavour-changing couplings will occur for charged leptons. In particular, those couplings proportional to the large topquark mass will dominate, unless they happen to be suppressed by small mixing angles. (iii) Our experience with the Kobayashi-Maskawa (KM) matrix suggests that the inter-generational mixing pattern for these flavour-changing interactions should be hierarchical. It would therefore follow that the large top-quark mass will have most influence on $\tau \rightarrow \mu$ conversions. Of course, a hierarchical mixing pattern is not inevitable, but at this juncture it nevertheless represents the best guess, in our opinion.

To get a feeling for the likely strength of these flavour-changing transitions, let us assume that all of the mixing matrices $V$ follow the qualitative form of the KM matrix, namely

$$
V \sim\left(\begin{array}{ccc}
1 & \epsilon & \epsilon^{3} \\
\epsilon & 1 & \epsilon^{2} \\
\epsilon^{3} & \epsilon^{2} & 1
\end{array}\right)
$$

where $\epsilon$ is a small parameter, which for the KM matrix equals about 0.2 . Note that this qualitative pattern is preserved when two such matrices are multiplied together. 
For purposes of illustration, if we take $\epsilon \simeq 0.1$, then for both up-quarks and charged leptons we find that

$$
V^{2} m^{\operatorname{diag}} V^{2} \sim\left(\begin{array}{ccc}
m_{1} & \epsilon m_{2} & \epsilon^{3} m_{3} \\
\epsilon m_{2} & m_{2} & \epsilon^{2} m_{3} \\
\epsilon^{3} m_{3} & \epsilon^{2} m_{3} & m_{3}
\end{array}\right)
$$

where $m_{1,2,3}$ refers to the first, second and third generation mass respectively. So, looking at charged-lepton transitions, we see that $\tau \rightarrow \mu$ is proportional to $\epsilon^{2} m_{t} \simeq 1$ $\mathrm{GeV}$. Observe that $\tau \rightarrow e$ is a further power of $\epsilon$ smaller, while $\mu \rightarrow e$ is driven by $\epsilon m_{c}$ which happens to be the same order of magnitude as $\tau \rightarrow e$.

Let us look now at the specific process $\mu^{-} \rightarrow e^{-} e^{+} e^{-}$, which will in general be mediated by all the neutral Higgs bosons $\eta$ and $h_{1,2}^{\prime}$. We will assume that all of the these Higgs particles have roughly the same mass $m_{\phi}$, and we will assume that no accidental cancellations occur between the three tree-level Feynman graphs contributing to this process. The partial decay width $\Gamma$ is then roughly given by

$$
\Gamma \simeq 10^{-4} \times\left(\frac{\epsilon m_{c} m_{u}}{m_{\phi}^{2}}\right)^{2} \frac{m_{\mu}^{5}}{u^{4}} .
$$

[This decay rate is calculated within Model 1. The Model 2 estimate is of exactly the same form, except that the $u$ quantity is replaced by $\left(u_{2}^{2}-u_{1}^{2}\right) / u$. If we take this quantity to have roughly the same value as $u$, then our semi-quantitative conclusions are the same for both models. Note also that this process requires one flavour-conserving vertex. The neutral Higgs boson Yukawa interactions feature both the $m_{u}$ and $m_{e}$ matrices for these flavour-conserving interactions. However, with the assumed mixing pattern and because $m_{u} \gg m_{e}$, we can approximately omit the piece proportional to the electron mass. Similar observations regarding the Model 2 estimate versus the Model 1 estimate, and the flavour-conserving vertex complication, will obtain for the other processes considered below.] The experimental bound is $\Gamma / \Gamma_{\mu}<10^{-12}$ [1], where $\Gamma_{\mu}$ is the total width of $\mu$, which leads to the constraint that

$$
\left(\frac{\epsilon m_{c} m_{u}}{m_{\phi}^{2}}\right)^{2}<10^{-11}
$$

having used $u \simeq 300 \mathrm{GeV}$. We see that typical values like $\epsilon \simeq 0.1$ and $m_{\phi} \simeq 300$ $\mathrm{GeV}$ fall well within this limit.

It is of interest to also look at $\tau$-lepton rare decays, since the large top-quark mass contributes. The bound for $\tau^{-} \rightarrow \mu^{-} \mu^{+} \mu^{-}$obtained from the experimental 
$\left.\operatorname{limit} \Gamma / \Gamma_{\tau}<10^{-5}[1]\right]$ is

$$
\left(\frac{\epsilon^{2} m_{t} m_{c}}{m_{\phi}^{2}}\right)^{2}<10^{-4}
$$

which again is easily satisfied with $\epsilon \simeq 0.1$ and $m_{\phi} \simeq 300 \mathrm{GeV}$. The other rare decays of the $\tau$ which can be mediated by tree-level Higgs-boson exchange, such as $\tau \rightarrow \mu e e$ and $\tau \rightarrow e e e$, all give much weaker constraints.

Turning now to the up-quark sector, we will examine $D^{0}-\bar{D}^{0}$ mixing and the rare decay $D^{0} \rightarrow \mu^{+} \mu^{-}$. Neutral Higgs boson exchange contributes to neutral $D$ meson mixing in both the s- and t-channels. This leads to an extra contribution to the mass difference given by

$$
\Delta m=\frac{1}{24}\left(\frac{\epsilon m_{\mu}}{u m_{\phi}}\right)^{2} m_{D} f_{D}^{2}\left[\frac{7 m_{D}^{2}}{\left(m_{u}+m_{c}\right)^{2}}+1\right],
$$

where $m_{D}=1.9 \mathrm{GeV}$ is the mass of the $D$-meson and $f_{D}=200 \mathrm{MeV}$ is its decay constant. We have used the standard vacuum-saturation approximation to calculate this expression. The experimental upper bound on the mass difference is $\Delta m<$ $10^{-13} \mathrm{GeV}$ [11], which leads to the limit

$$
\left(\frac{\epsilon m_{\mu}}{m_{\phi}}\right)^{2}<10^{-7}
$$

Again, the values $\epsilon=0.1$ and $m_{\phi}=300 \mathrm{GeV}$ easily satisfy this constraint.

The partial width of the decay $D^{0} \rightarrow \mu^{+} \mu^{-}$is calculated within the vacuum saturation approximation to be

$$
\Gamma_{\mu^{+} \mu^{-}}=\frac{1}{8 \pi}\left(\frac{\epsilon m_{\mu} m_{c}}{u^{2} m_{\phi}^{2}}\right)^{2}\left(\frac{f_{D}}{m_{u}+m_{c}}\right)^{2} m_{D}^{5} .
$$

The experimental bound is $\Gamma_{\mu^{+} \mu^{-}}<10^{-17} \mathrm{GeV}$ [11], which leads to the constraint

$$
\left(\frac{\epsilon m_{\mu} m_{c}}{m_{\phi}^{2}}\right)^{2}<10^{-5}
$$

Once again, this is easily satisfied with $\epsilon=0.1$ and $m_{\phi}=300 \mathrm{GeV}$.

So, we have shown that the tree-level flavour-changing neutral Higgs boson effects within the model can easily fall within current experimental limits on unseen decays and on neutral meson mixing. Most spectacularly, the down-quark sector yields no bounds because the neutrinos are constrained to be light. But, as we have 
just seen, the charged-lepton and up-quark sector bounds can be met with Higgs boson masses of the order of the electroweak scale, provided we invoke hierarchical generation mixing. Note, however, that the mixing hierarchy need be no more severe than what we find in the KM matrix.

\subsubsection{Higher loop effects}

The main phenomenological point we want to make in this paper is that the existence of neutral flavour-changing vertices in quark-lepton symmetric models does not necessarily mean that the offending electroweak Higgs bosons have to be made artificially heavy. We feel this point is adequately demonstrated by the bounds calculated in the preceeding subsubsection for tree-level processes. However, there are a whole host of interesting effects that will be induced at higher-loop levels also, and so for completeness we include a brief discussion of some of them.

For instance, radiative decays of the second and third generation quarks and leptons will be induced at 1-loop order. In addition to the SM graph featuring a virtual fermion/ $W$ loop, there will be new contributions coming from charged-Higgsboson/fermion loops and flavour-changing neutral-Higgs-boson/fermion loops. (There are also small 1-loop contributions from a heavy- $W^{\prime} /$ lipton virtual pair. See Ref. [2] for a brief discussion.) It would be interesting to compare the predictions for these decays in $q-\ell$ symmetric models with other two-Higgs-doublet models [12]. For instance, in Model 1 with zero Dirac neutrino masses there will be no contribution from either charged or neutral Higgs bosons to $b \rightarrow s \gamma$. This amusing fact may be important, given that the recent CLEO measurement of $B \rightarrow K^{*} \gamma$ is consistent with SM expectations [13]. Similar 1-loop effects will also contribute to processes like $b \rightarrow s \ell^{+} \ell^{-}$where $\ell$ is a lepton.

Charged Higgs bosons will contribute to neutral-meson mixing through box graphs, and the distinctive $\mathrm{q}-\ell$ partnership phenomenon in the Yukawa Lagrangians should produce interesting systematics. For instance, in Model 2 the charged Higgs bosons have couplings proportional to both quark and lepton mass matrices, whereas in Model 1 the coupling is exclusively through the partner lepton mass matrix. It would be interesting to see how expectations for both of these models compare with expectations in other two-Higgs-doublet models [12].

Finally, we note that there will be extra contributions to the anomalous moments of charged leptons at 1-loop level. For instance, both the charged and neutral Higgs

bosons contribute to the anomalous magnetic moment $a_{\mu}$ of the muon. What is of some interest here is that the vertices involved will have pieces proportional to the 
up-quark masses, and so will be larger than those in the usual two-Higgs-doublet models. Given our assumed mixing pattern, $a_{\mu}$ will be about equally affected by

$m_{c}^{2}$ and $\left(\epsilon m_{t}\right)^{2}$, which are both about two orders of magnitude greater than the corresponding quantity in the SM, namely $m_{\mu}^{2}$. The SM neutral Higgs contribution gives roughly $a_{\mu} \simeq 10^{-13}$ [14, so that the contribution in $\mathrm{q}-\ell$ symmetric models is roughly $10^{-11}$. This is comfortably below the experimental error on $a_{\mu}$ which is about $10^{-8}$.

\subsubsection{The heavy Higgs bosons}

We end this section with a few words about the phenomenology of the heavy Higgs bosons in Higgs sector $\mathrm{B}$, namely the neutral field $H$ and the charged and coloured field $\chi_{1}$.

Particle $H$ is expected to be heavy because it is associated with leptonic colour and discrete symmetry breaking. However, it couples only very weakly to standard particles and is thus very difficult to produce in the laboratory. Its only direct Yukawa couplings are to liptons, not leptons, and it does not couple to any of the electroweak gauge bosons. Its tree-level couplings to standard particles occur therefore only through mixing with $h_{1,2}^{\prime}$, which we expect to be quite suppressed. We thus expect the actual phenomenological bound on $H$ to be very weak, although the generic expectation is that it ought to be relatively heavy.

The coloured scalar $\chi_{1}$ contributes to neutral meson mixing, as discussed in Ref. [15]. The generic bound obtained is that $h_{2} / m_{\chi}<10^{-3} \mathrm{GeV}^{-1}$, where $h_{2}$ is relevant Yukawa coupling constant. We note that this is a weak constraint.

\section{Conclusion}

The simplest realistic Higgs sector in models with leptonic colour and a quarklepton discrete symmetry contains two electroweak Higgs doublets. We have demonstrated that the effective two-Higgs-doublet model obtained at the electroweak scale is unusual and interesting, because Yukawa coupling constants are in important instances proportional to the mass matrix of the discrete symmetry partner of the fermion in question, rather than of the fermion itself. In particular, the flavourchanging neutral vertices are always proportional to the mass of the partner fermion. If neutrinos are Dirac particles then this implies that flavour-changing neutral Higgs effects in the down-quark sector are negligible, and so all of the traditional con- 
straints like those from neutral kaon mixing are absent. The largest flavour-violating effects occur in the charged-lepton and up-quark sectors, but we showed that these effects are typically undetectable if a Kobayashi-Maskawa pattern of inter-generation mixing is invoked. We conclude, therefore, that electroweak Higgs bosons with masses in the $100-\mathrm{GeV}$ range are perfectly acceptable, even though some of them mediate neutral flavour-changing processes at tree-level.

\section{Acknowledgements}

YL wishes to thank Xiao-Gang He and Andrew J. Davies for very useful discussions. RRV would like to thank Henry Lew and Robert Foot for interesting communications and Xiao-Gang He for useful discussions. He is supported by grants from the University of Melbourne.

\section{References}

[1] R. Foot and H. Lew, Phys. Rev. D41, 3502 (1990).

[2] R. Foot, H. Lew and R. R. Volkas, Phys. Rev. D44, 1531 (1991).

[3] R. Foot, H. Lew and R. R. Volkas, Phys. Rev. D44, 859 (1991) and (E) ibid. D47, 1272 (1993); G. C. Joshi and R. R. Volkas, Phys. Rev. D45, 1711 (1992).

[4] R. Foot and H. Lew, Mod. Phys. Lett. A7, 301 (1992).

[5] H. Lew and R. R. Volkas, Phys. Rev. D47, 1356 (1993).

[6] R. Foot, H. Lew and R. R. Volkas, Int. J. Mod. Phys. A8, 983 (1993).

[7] For studies of glueballs in a related model see E. D. Carlson et al., Phys. Rev. D44, 1555 (1991) and R. Foot and H. Lew, Purdue University Report No. PURD-TH-92-3 (unpublished).

[8] M. Gell-Mann, P. Ramond and R. Slansky, in Supergravity, eds. P. Van Nieuwenhuizen and D. Z. Freedman (North-Holland, Amsterdam, 1979); T. Yanagida in Proc. Workshop on Unified Theory and Baryon Number of the Universe, eds. A. Sawada and H. Sugawara (KEK, Tsukuba-Gun, Ibaraki-Ken, Japan, 1979); R. N. Mohapatra and G. Senjanovic, Phys. Rev. Lett. 44, 912 (1980). 
[9] R. Foot, H. Lew and R. R. Volkas, University of Melbourne Report No. UMP-91/46 (Mod. Phys. Lett., in press).

[10] R. Foot and H. Lew, Nuovo Cim. A104, 167 (1991).

[11] Particle Data Group, Phys. Rev. D45, S1 (1992).

[12] For a review see J. F. Gunion, H. E. Haber, G. Kane and S. Dawson, Higgs Hunter's Guide (Addison-Wesley, 1990, Redwood City).

[13] CLEO Collaboration, forthcoming report.

[14] J. P. Leveille, Nucl. Phys. B137, 63 (1978).

[15] A. J. Davies and X.-G. He, Phys. Rev. D43, 225 (1991). 